\title{
Síndrome de la Arteria Mesentérica Superior Presentación de Dos Casos
}

\author{
Dra. Gloria Ríos M. 1; Dr. Jurgen Thümler U. 2; Dr. Carlos Toro A. ${ }^{3}$
}

Superior Mesenteric Artery Syndrome

\begin{abstract}
Two adolescent girls with evidence of extrinsic duodenal obstruction caused by superior mesenteric artery are presented. Both cases shared in common low food ungestion of psycological origin, but their initial complaints were different: the first patient was refered because of intermitent abdominal pain and vomitus, the second one was admited for acute high intestinal oclussión. Both girls envolved favourably under therapy with small frecuent meals and postural treatment.

(Key words: Duodenal obstruction. Superior mesenteric artery).
\end{abstract}

La primera descripción del sindrome de la arteria mesentérica superior (S.A.M.S.) parece corresponder a Boener en 1754. Rokitansky en 1861 sugirió el mecanismo de su producción $y$ Wilkie en 1921, publicó la primera serie de 75 casos de "compresión duodenal por la arteria mesentérica superior", realizando un acabado estudio sobre el tema, por esta razón, se conoce también como síndrome de Wilkie ${ }^{1.2}$.

Este síndrome es una forma de obstrucción intestinal alta debida a compresión de la tercera porción del duodeno por la AMS contra la aorta y la columna vertebral. La obstrucción resultante puede ser aguda o crónica, parcial e intermitente $\mathrm{e}^{3}$.

Nomalmente la AMS emerge de la aorta en sentido caudal, a la altura de la primera vértebra lumbar $\left(L_{1}\right)$ en un ángulo de 45 a 60 grados. El duodeno pasa por detrás de la arteria frente a $\mathbf{L}_{3}$. En el individuo normal, la grasa retroperitoneal separa la AMS de la aorta y la columna vertebral impidiendo que comprima al duodeno $1,4,5$ (Fig. 1).

El síndrome AMS es una causa rara de obstrucción intestinal alta en niños. Muchas veces el diagnóstico es difícil, especialmente cuando se

1. Médico. Unidad de Gastroenterología. Servicio de Pediatría. Hospital Exeyuiel Gonzalez Cortés. S.S.M.S. Santiago.

2. Médico. Servicio de Radiologia. Hospital Exequiel Ganzález Cortés. SM.S.S. Santiago.

3. Médico. Profesor asociado de Pediatría. Depto. de Pediatría. División Ciencias Médicas Sur. Facultad de Medicina. Universidad de Chile. trata de obstrucción intestinal intermitente. A continuación se presentan 2 casos cuyas manifestaciones clínicas fueron distintas entre si, con el propósito de estimular su búsqueda en situacio. nes similares.

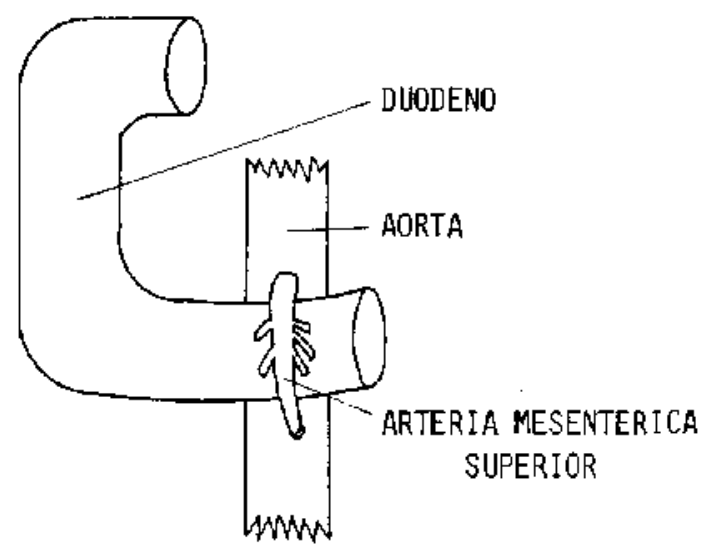

Figura 1: Representación esquemática de las relaciones de la ar teria mesentérica superior con la tercera porción del duodeno.

\section{Casos Clínicos}

Caso 1: ARQ. Niña de 13 años de edad, sufrió dos años antes, un trastorno conductual caracterizado por rechazo de su propia imagen y de los cambios corporales de la pubertad, asociado con escaso aumento de peso sin compromiso de la talla. Durante el año que precedió a su consulta tuvo episodios aislados de dolor abdominal djfuso de carácter cólico que relacionaba, en algu- 
nas oportunidades, con la ingestión de alimentos grasos. Consultó por crisis de dolor, cólico, periumbilical, acompañado de vómitos biliosos profusos, que la llevaron a la deshidratación. E] dolor no tenía factor desencadenante, aliviaba parcialmente con los vómítos y su respuesta a los antiespasmódicos era errática; cada crisis duraba dos o cuatro días con periodos intercríticos de siete a quince días. Evolucionó con descenso progresivo de peso y recuperaciones parciales de éste entre las crisis. En uno de los episodios se constató retención gástrica de $700 \mathrm{cc}$ de líquido bilioso. Hemograma, velocidad de eritrosedimentación (VES) amilasemia, ami]asuria, creatinjnemia, fosfatasa alcalina, transaminasas, protejnemia, PPD, sedimento de orina y urocultivo, radiografías de esófago, estómago y duodeno (R.E.E.D.) en periodos intercríticos, ecografia abdominal y endoscopía digestiva alta normales. Los estudios destinados a investigar intoxicación por plomo y alteración en el metabolismo de las porfirinas fueron infructuosas. En las deposiciones se encontraron quistes de Giardia lamblia; concomitantemente las radiografías contrastadas del intestino delgado mostraban floculación del medio de contraste; carotinemia 40 ug $\%$ de $\beta$ caroteno, volviendo a cifras nomales después del tratamiento de la infección por Giardia lamblia con Metronidazol. Sin embatgo, la paciente continuó con las mismas molestias. Durante una crisis de dolor abơominal se repitió la R.E.E.D. que esta vez mostró obstrucción extrinseca al paso del bario en la porción distal del duodeno, que cedía parcialmente con los cambios de posición (Fig. 2). Se trató con hiperalimentación enteral fraccionada y posición ventrolateral izquierda observando remisión de los sintomas $y$ ascenso ponderal progresivo. Ha sido controlada durante 18 meses, permaneciendo asintomática. Tiene 14,5 años, pesa $42 \mathrm{~kg}$ y mide $148 \mathrm{~cm}$.

Caso 2: MMR. Niña de 13 años, por iniciativa propia siguió un estricto régimen hipocalórico logrando un descenso importante de peso, sin disminuir sus actividades. Sus molestias comenzaron bruscamente con vómitos frecuentes $y$ abundantes post prandiales precoces, conteniendo alimientos y bilis, asociados con astenia y adinamia progresiva. Ingresó al hospital en estado de caquexia (Peso: $29.800 \mathrm{gr}$; Talla $152 \mathrm{~cm}$ ) estuporosa, con evidencias de deshidratación, abdomen excavado y una gran prominencia en el epigástrico que desaparecieron cuando se extrajeron $1.500 \mathrm{ml}$ de líquido retenido en el estómago. Hemograma, VES, PPD, radiografía de tórax, glicemia, amilasemia, amilasuria, transaminasas, fosfatasa alcalina normales. En la radiografía de

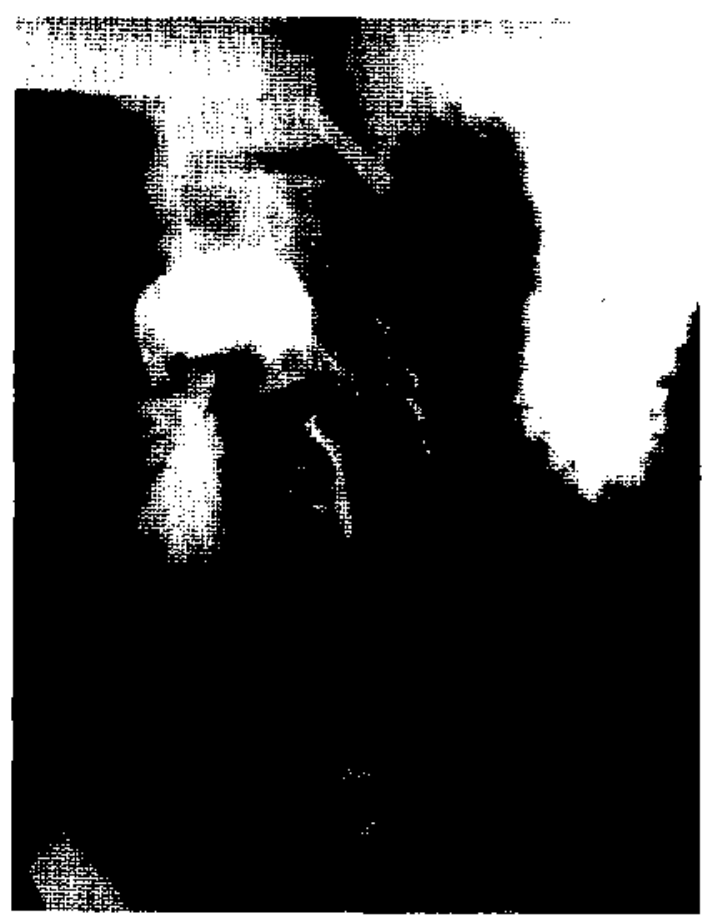

Figura 2: Obstrucción al paso del bario en la tercera porción del duodeno con dilatación proximal.

abdomen simple había imagen de "doble burbuja", gástrica y duodenal; que en R.E.E.D., coincidía con la dilatación de ambos segmentos digestivos y obstrucción total del paso de bario en el duodeno (Fig. 3).

Se trató con hiperalimentación enteral por yeyunotomía; recuperó progresivamente el peso, desapareció paulatinamente la retención gástrica y los vómitos y fue posible reiniciar la alimentación oral. A los 45 días de tratamiento la R.E.E.D. mostraba persistencia de ondas peristálticas y antiperistálticas con buen paso del medio de contraste hacia el duodeno, siendo este más efectivo en decúbito ventral. Ha cumplido 5 meses de seguimiento, permanece asintomática, pesa $37,3 \mathrm{~kg}$ y mide $153 \mathrm{~cm}$.

\section{DISCUSION}

Ha sido difícil determinar ]a real incidencia de este síndrome porque se le dan distintos nom. bres, no es fácil de reconocer y se presenta de diferentes maneras $5,6,7$. En adultos es más frecuente en enfermos crónicos portadores de afecciones que producen caquexia ${ }^{8}$. En niños se ve preferentemente en casos con grandes quemaduras que bajan bruscamente de peso, también en pacientes inmovilizados en hiperextensión dorsal, como parte de tratamiento de escoliosis o frac- 

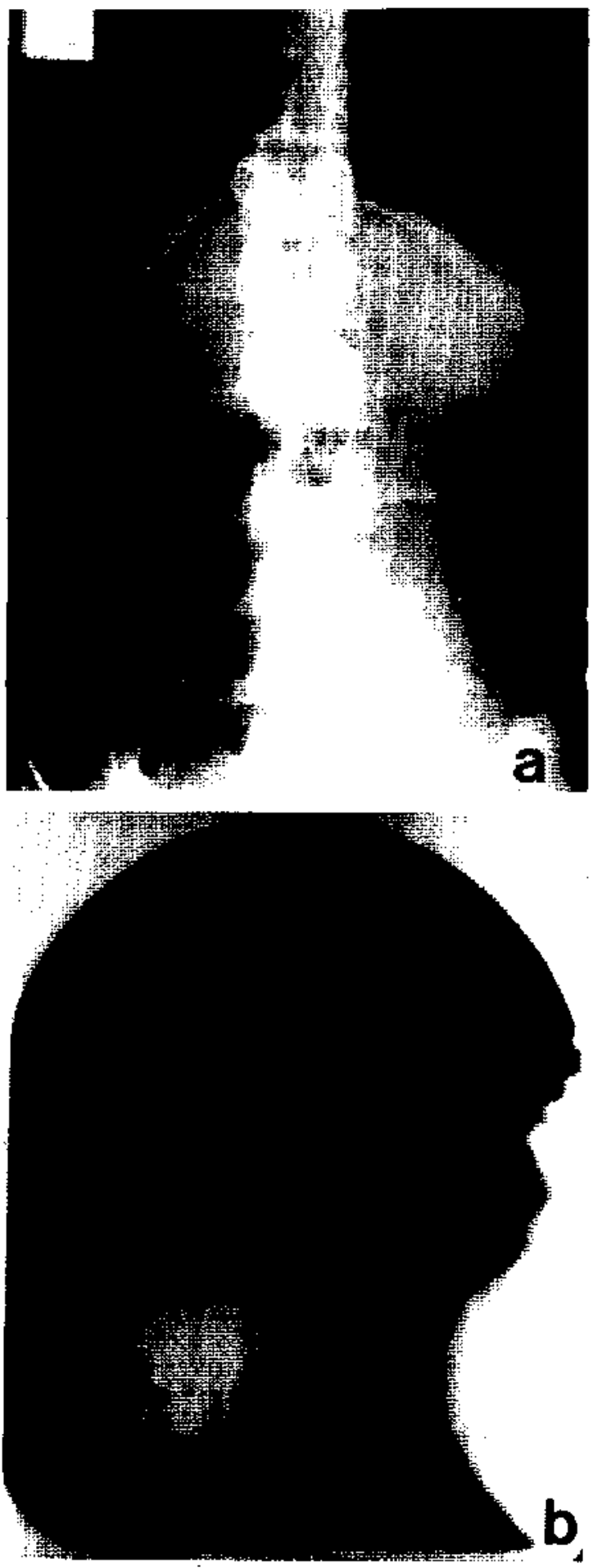

Figura 3: a) Imagen de doble burbuja gastro-duodenal en la radiografía simple de abdomen. b) Tránsito gastroduodenal. A la derecha se observa la imagen gástrica. A la izquierda, abajo, dilatación duodenal proxinal a un obstáculo al paso del bario.

turas vertebrales; por esto se le conoce también como "enfermedad del yeso": en esta situación la hiperextensión determina aumento en la ten- sión de la raíz del mesenterio facilitando la compresión del duodeno por la arteria ${ }^{3,5}$. Otro grupo de pacientes está constituido por niños que pierden peso bruscamente o crecen rápidamente sin incrementos ponderales; en ellos la causa de] trastorno sería la disminución de la grasa retroperitoneal permitiendo que la arteria comprima el duodeno $4,7,9$. Nuestros casos corresponden a este último grupo. La primera paciente aumentó de talla sin aumento de peso concomitante y la segunda presentó descenso de peso exclusivamen. te.

Como no todos los niños con las características señaladas desarrollan el sindrome, se ha planteado que quienes lo hacen podrían tener factores anatómicos predisponentes, entre ellos: anomalías en la altura y tensión del ligamento de Treitz, posición más alta de la tercera porción del duodeno, origen anómalo de la AMS y anomalias en la relación de la aorta con los cuerpos vertebrales 1 .

Las manifestaciones caracteristicas son: dolor abdominal post prandial precoz, sensación de plenitud gástrica y vómitos biliosos. Muchos pacientes aprenden que la sensación de plenitud precede al dolor y los vómitos y que éstos no se presentan si dejan de comer cuando aquella es percibida. Así, los pacientes limitan la ingestión de alimento, manteniendo el déficit de peso, situación que puede ser confundida con anorexia nerviosa ${ }^{5,9}$ : estos pacientes pueden ser derivados, en primera instancia, al psiquiatra retardándose indebidamente el diagnóstico. También pueden coexistir ambas enfermedades. En nuestra segunda paciente se encontraron síntomas compatibles con anorexia nerviosa como el deseo patológico de delgadez llegando a la caquexia a pesar de lo cual desempeñaba sin inconvenientes las actividades escolares ${ }^{10}$

Cuando la obstrucción duodenal es parcial e intermitente, la consulta es por crisis de dolor abdominal y vómitos biliosos, como en nuestro primer paciente, cuyo SAMS probablemente comenzó después de haber crecido sin aumentar adecuadamente de peso $y$ se manifestó durante un afro como dolor abdominal recurrente, que en algunas oportunidades fue calificado de "cólico biliar" pues parecía guardar relación con la ingestión de alimentos grasos. Esta forma de presen. tación puede sugerir una colecistopatía $y, m u-$ chas veces estos pacientes son referidos al psiquiatra porque no se demuestra en la colecistografía anormalidad de las vias biliares ${ }^{9}$.

En el examen físico destaca la disminución del paniculo adiposo. Puede observarse abomb aniento epigástrico $\mathrm{y}$ ondas peristálticas in testinales. $\mathrm{E} 1$ signo más especifico es la auscultación de un soplo sistólico en el epigastrio, que característica- 
mente no es transmitido desde el área cardiaca y desaparece al cambiar la posición de decúbito supino a prono. ${ }^{11}$

E] diagnóstico se confírma mediante la radiología. En los casos de obstrucción total, la radio. grafía de abdomen simple puede mostrar una imagen de doble butbuja que recuerda el aspecto de la estenosis duodenal congénita ${ }^{4}$. Las imágenes que configuran los criterios radiológicos para hacer el diagnóstico son: dilatación de la primera y segunda porción del duodeno con o sin dilatación gástrica compresión abrupta vertical $y$ oblicua de los pliegues de la mucosa de la segunda porción del duodeno; flujo antiperistáltico de bario proximal a la obstrucción; retardo del tránsito en la región gastroduodenal de 4 a 6 horas; alivio de la obstrucción con los cambios de posición del paciente?

El tratamiento es conservador $5,7,9$ consiste en hiperalimentación para lograr aumento del peso y recuperar la grasa retroperitoneal con la consiguiente reconstitución de la anatomía. La alimentación puede ser fraccionada y por vía oral o por sonda nasoyeyunal.

Nuestras pacientes fueron tratadas con hiperalimentación fraccionada, posición ventrolateral izquierda para facilitar el vaciamiento gástrico, obteniendo regresión del dolor y retención gástrica y ascenso ponderal progresivo. Cuando el paciente logra un ascenso ponderal de $2.5 \mathrm{~kg}$ asociado con comprensión del mecanismo que desencadenó el trastorno, quedaría capacitado para regular su hábito de alimentación y continuar su recuperación ${ }^{5}$.

Cuando fracasa el tratamiento médico estaría indicada la corrección quirúrgica de la obstrucción para la cual se han descrito tres técnicas: gastroyeyunoestomia, duodenoyeyunostomía y operación de Strong, donde se secciona el ligamento de Treitz y se moviliza el duodeno con transposición de colon derecho ${ }^{7}$. Esta última seria la técnica de elección en pacientes que no han sido operados previamente.

\section{RESUMEN}

Se presentan dos niñas con síndrome de arteria mesentérica superior $y$ distintas formas de presentación. El primer caso consultó por crisis de dolor abdominal y vómitos que se debian a obstrucción crónica e intermitente del duodeno por la arteria mesentérica. El segundo caso comenzó como obstrucción intestinal alta aguda. Ambas respondieron bien al tratamiento médico con hiperalimentación fraccionada, decúbito lateral derecho post prandial y apoyo psicológico.

\section{REFERENCIAS}

1. Gray S.W., Skandalakis J.E.; A nomalías congénitas. Ed. Pediátrica 1975, Barcelona, págs. 195-19?.

2. Lundell L., Thulin A.: Wilkic's yndrome - a rarity? : BI. J. Surg. 67: 604, 1980.

3. Gondos B.: Duodenal compression defect and the "superior mesenteric artery syndrome" Radiology $123: 575,1977$.

4. Burrington L., Wayne E.: Obstruction of the duodenum by the superior mesenteric artery - Does it exist in children'? J. Pedlatr. Surg. 9: 733, 1974.

5. Burrington J.: Superior mesenteric artery in childten. Am. J. Dis. Child. 130: 1367, 1976.

6. Feiss $J$., Goldenberg A., Plevy D. Luckman G.: Superior mesenteric attery syndrome simulating acute pancreatitis. Am. J. Gastroenterol. 66: 476, 1976.

7. Hines $J$. Gore R., Ballantyne G.: Superior mesenteric artery syndrome. Diagnostic criteria and therapeutic approaches. Am. J. Surg. 148: 630, 1984.

8. Lee C.S., Mangla J.: Superior mesenteric artery compression syndrome. Am. J. Gastroenterol. 70: $141,1978$.

9. Shandling B. er al: The so-called superior mesenteric artery syndrome. Am. J. Dis. Child. 130: $1371,1976$.

10. Vivanco N., Pumarino H.: Diagnóstico de la anoraxia nerviosa: una sistematización de sus manifestaciones. Tipos, variedades y diagnóstico diferencial. Bol. Hosp. S.J. de Dios 32: 312, 1985.

11. Barnet $R$.: A simple diagnostic sign in the superior mesenteric artery syndrome in a burned patient. Br. J. Plastic. Surg. 29:322, 1976. 